\title{
Research on Stability of Gray Value of Excited-State Fluorescent Oil Film Based on Variable Light Vector Angle
}

\author{
Hongjiang Qian $\mathbb{D}^{1},{ }^{1}$ Xiucheng Dong $\mathbb{D},{ }^{1}$ and Zhengyu Zhang $\mathbb{D}^{2}$ \\ ${ }^{1}$ College of Electrical Engineering and Electronic Information, Xihua University, Chengdu 610039, China \\ ${ }^{2}$ High Speed Aerodynamics Institute, China Aerodynamics Research and Development Center, Mianyang 621000, China \\ Correspondence should be addressed to Xiucheng Dong; 2778805412@qq.com
}

Received 5 July 2021; Revised 25 August 2021; Accepted 17 November 2021; Published 21 December 2021

Academic Editor: Qilong Xue

Copyright (C) 2021 Hongjiang Qian et al. This is an open access article distributed under the Creative Commons Attribution License, which permits unrestricted use, distribution, and reproduction in any medium, provided the original work is properly cited.

\begin{abstract}
In global skin-friction measurement of aircraft, the fluorescent oil film method can characterize the distribution of skin friction well. However, in an actual wind tunnel test, the wing of the aircraft will inevitably produce corresponding vibrations due to the influence of wind, which will change the relative position between fluorescent oil film and UV (ultraviolet) excitation light source (position fixed). This also directly affects gray value imaging of fluorescent oil films. Based on this, a mathematical model is established to judge the stability of the gray value of fluorescent oil film in this vibrational environment; then, the model can be solved to obtain the vibrational range constraint that enables the gray value of fluorescent oil film to be stabilized. In order to simplify the calculation process, the light vector angle is used to describe the constraint, which also makes the results more intuitive. Through experimental analysis and demonstration, the prediction accuracy of this model can reach $95.61 \%$, which has certain practical engineering application significance.
\end{abstract}

\section{Introduction}

Skin friction is one of the most important physical quantities in aerodynamics, as an essential component of the total resistance in an aircraft. It can describe the state of the turbulent boundary layer well and is also one of the most difficult physical quantities to determine [1-4]. According to available research, the skin friction of modern civil aircraft can account for about $50 \%$ of total resistance during stable operation [5-8]. It holds a dominant position when compared to other resistance factors. Therefore, it has great military and economic significance to reduce skin friction in terms of saving fuel consumption and increasing the payload of the aircraft $[9,10]$.

Traditional methods of measuring skin friction are mainly local indirect methods, such as hot-wired method, hot-film anemometry, Preston tube, Stanton tube, boundary-layer separation, and laser Doppler anemometry [11]. Subsequently, Professor Tianshu Liu from Western Michigan University proposed a method combining fluorescent oil film and machine vision to directly measure skin friction in 2008 [12], which has the advantages of simple equipment operation, high spatial resolution, no special requirements for measurement tool, fast calculation speed, and high calculation accuracy compared to traditional measurement methods. Now, this method has become a mainstream method for the direct measurement of skin friction in the international arena. In 2011, Peng and Xiao revealed that the gray value of fluorescent oil film was linearly related to the thickness through experiments and introduced this linear equation into a relative skin-friction resistance model [13] so as to obtain a mapping model between relative skin-friction resistance of the imaging plane and the gray value of fluorescent oil film. The exploration of this model provided strong support for studying and solving the direct measurement of skin-friction resistance. In 2016, Zhan et al. [14] conducted a systematic review and analysis of the fluorescent oil film control equations and then combined the optical flow method in the experiment to verify the feasibility of the fluorescent oil film control model. In the most recent year of 
2019, Zou et al. [15] used the image correlation method to obtain adjacent time-series images and the mapping matrix of their background textures, based on the continuity principle of model motion, and the global optimization equations of the mapping matrix were solved. Then, the optical flow method was used to decouple the model vibration from the path motion of the fluorescent oil film surface, which improved the accuracy of the skin-friction resistance solution.

However, the linear model of gray value and fluorescent oil film thickness mentioned in many of the above studies do not take into account other disturbing factors such as the vibrational motion of the wing. For example, assuming that when UV (ultraviolet) excitation light source is fixed, the thickness of fluorescent oil film on the wing is constant and does not change with time. The aircraft wing will move with small vibrations in the actual wind tunnel test and high wind speeds, which will cause the fluorescent oil film attached to the wing to move with it too. On the basis of this movement, the spatial position of fluorescent oil film will change. This variation equates to a change in the spatial vector between UV excitation light source and fluorescent oil film. The spatial vector contains information on the distance and spatial orientation from UV excitation light source to fluorescent oil film. It changes the intensity of the light produced when the fluorescent oil film is excited by the UV excitation light source. It is well known that the gray value imaging of fluorescent oil film is linearly related to the intensity of UV light that excites the fluorescent oil film. The light intensity of the fluorescent oil film is closely related to the distance between the UV excitation source and the fluorescent oil film, that is, a longer distance of fluorescent oil film from UV excitation light source, the lower light emitted from fluorescent oil film, and the darker gray value presented. In order to facilitate the calculation of the model, the vector angle and distance factor of UV light are introduced to represent the variable space vector between fixed UV excitation light source and fluorescent oil film, using the light vector angle to characterize the wing vibration as a constraint. So a mathematical model between the light vector angle and gray value is then established to determine whether the gray value of the fluorescent oil film is stable within a certain range of light vector angle variations.

In order to solve the above-related problems, this paper analyzes and investigates the stability of the gray value of fluorescent oil film in a vibrational environment. The variational range constraint that stabilizes the gray value of the fluorescent oil film is explored, and the light vector angle is used to characterize this vibrational range constraint, which makes the results more intuitive. Based on the vibrational environment, a theoretical analysis on the stability of gray value is carried out, and we establish a mathematical model from the light vector angle and gray value. A range of light vector angular variation (maximum oscillation range) that stabilizes the gray value of the fluorescent oil film is also researched. As shown by experimental results, the measurement accuracy of the theoretical mathematical model can reach $95.61 \%$, which has certain practical engineering application significance. At the same time, the stability of the gray value of fluorescent oil film has been studied to limit the interference factors in the measurement of skin friction resistance and to provide a range of applications for the use with fluorescent oil films.

\section{Mathematical Model Theory}

Assuming that the fluorescent oil film is with high viscosity coefficient, the effects of wing vibration or other disturbing factors on the film thickness are ignored, and only these effects on the gray value of fluorescent oil film are considered. A mathematical model is developed in these above contexts. Then, the camera is calibrated to obtain the internal and external parameters. The position pose is calculated to obtain matrix $R$ (rotation) and matrix $T$ (translation) for the transformation of the pixel coordinate system to the world coordinate system. This method enables the world coordinate position corresponding to the pixel coordinate point to be found accurately. By obtaining information on the spatial vector variation between the fluorescent oil film and the fixed UV excitation light source, UV vector angles are used to describe the range of spatial vector variations which facilitate model calculations. Finally, a complete mathematical model for judging the stability of the gray value has been established. The vibration of the simulated wing is shown in Figure 1. The mathematical theory is derived as follows. This world coordinate system is established with a fixed UV excitation light source as the initial coordinate point, which is shown in Figure 2.

Let the UV excitation light source be $I_{0}$, the fluorescence brightness at coordinate point 1 named " $I_{1}$," and the fluorescence brightness at coordinate point 2 after the offset named " $I_{2}$," whose coordinate information is shown in the following equation:

$$
\left\{\begin{array}{l}
I_{0}(0,0,0), \\
I_{1}(x, y, z), \\
I_{2}(x+\Delta x, y+\Delta y, z+\Delta z) .
\end{array}\right.
$$

Here, $\Delta x, \Delta y, \Delta z$ are the offsets of $x, y, z$. From this, the vector modulus lengths of $I_{1}, I_{2}$, and $I_{0}$ named " $\mid \overrightarrow{R_{1} \mid}$ and $\left|\overrightarrow{R_{2}}\right|, "$ and the modulus length of the offset vector named " $\overrightarrow{\Delta \mid}, "$ as shown in the following equation:

$$
\left\{\begin{array}{l}
\overrightarrow{\left|R_{1}\right|}=\sqrt{\left[\vec{R}_{(x, y, z)}\right]^{2}} \\
\overrightarrow{\left|R_{2}\right|}=\sqrt{\left[\vec{R}_{(x, y, z)}+\vec{\Delta}_{(\Delta x, \Delta y, \Delta z)}\right]^{2}} \\
\overrightarrow{|\Delta|}=\sqrt{\left[\vec{\Delta}_{(\Delta x, \Delta y, \Delta z)}\right]^{2}}
\end{array}\right.
$$

The brightness equation of the fluorescent oil film [11] is then given by

$$
I_{(n)}=c \cdot \phi(x, y, z, t) \int_{0}^{h} I_{0(x, y, z)} \mathrm{d}_{z} .
$$

Here, the absorption efficiency coefficient of the fluorescent oil film named " $c$," and the molecular coefficient of the 
fluorescent oil film concentration named " $\phi(x, y, z, t)$." If $I_{0(x, y, z)}$ is the brightness of UV excitation light source, then $I_{(n)}$ is the brightness of the excited fluorescent oil film. And then, the thickness of the fluorescent oil film named " $h$. "

By rearranging, we observe that the brightness equation may be expressed as equation (4). The brightness value of the fluorescent oil film at $I_{1}$ and $I_{2}$ can be obtained, and we designate the light intensity of the UV excitation light source relative ${ }_{\wedge}$ to the point $(x, y, z)$ as $I_{0(x, y, z)}$; similarly, $I_{0(x+\Delta x, y+\Delta y, z+\Delta z)}$ is the light intensity of the UV excitation light source relative to the point $(x+\Delta x, y+\Delta y, z+\Delta z)$.

$$
\left\{\begin{array}{l}
I_{1}=c \cdot \phi(x, y, z, t) \int_{0}^{h} I_{0(x, y, z)} \mathrm{d}_{z}, \\
I_{2}=c \cdot \phi(x+\Delta x, y+\Delta y, z+\Delta z, t) \int_{0}^{h} I_{0(x+\Delta x, y+\Delta y, z+\Delta z)} \mathrm{d}_{(z+\Delta z)} .
\end{array}\right.
$$

According to research literature, the power of UV excitation light source [16-18] is shown as

$$
I_{0}=\frac{W}{4 \pi \cdot d^{2}} .
$$

The power parameter of the UV excitation light source is $W$, and there a distance is $d$. The relationship between $I_{1}$ and $I_{2}$ is further processed, as shown in the following equation:

$$
\frac{I_{1}}{I_{2}}=\frac{c \cdot \phi_{(x, y, z, t)} \int_{0}^{h} \hat{I}_{0(x, y, z)} \mathrm{d}_{z}}{c \cdot \phi_{(x+\Delta x, y+\Delta y, z+\Delta z, t)} \int_{0}^{h} \hat{I}_{0}(x+\Delta x, y+\Delta y, z+\Delta z)} \mathrm{d}_{(z+\Delta z)},
$$

Assuming that the concentration of the fluorescent oil film is uniform everywhere, then the thickness of fluorescent oil film is constant. So these can be written as

$$
\begin{aligned}
\phi_{(x, y, z, t)} & =\phi_{(x+\Delta x, y+\Delta y, z+\Delta, t)}, \\
\frac{\partial h}{\partial t} & =0 .
\end{aligned}
$$

Then, we can get the luminance ratio relationship as

$$
\frac{I_{1}}{I_{2}}=\frac{{\overrightarrow{R_{2}}}^{2}}{\left|\vec{R}_{1}\right|} \text {. }
$$

The fluorescence brightness at $I_{1}$ is subtracted from the fluorescence brightness at $I_{2}$ to obtain the difference $\Delta S$. It can be defined as

$$
\Delta S=I_{1}-I_{2}=\left(1-\frac{{\overrightarrow{\mid R_{2}}}^{2}}{\left|R_{1}\right|}\right) \cdot I_{1} .
$$

in order to explore the stability of the gray value under the condition of wing vibration or other disturbance conditions. In other words, it can be said as the maximum range of change in light vector angle caused by wing vibration or other disturbance when the gray value is stable. Therefore, a constraint is established to indicate that the gray value of the fluorescent oil film is stable, as shown in the following equation:

$$
0 \leq \Delta S<1
$$

Then, the equation can be expanded as follows:

$$
\begin{aligned}
\Delta S & =\left(1-\frac{\mid{\overrightarrow{R_{2}}}^{2}}{\mid{\overrightarrow{R_{1}}}^{2}}\right) \cdot I_{1}<1 \\
& =\frac{\left[\overrightarrow{R_{1}}(x, y, z)\right.}{\left[\vec{R}_{(x, y, z)}\right]^{2}}<\frac{I_{1}}{I_{1}-1} .
\end{aligned}
$$

It can be rewritten as

$$
\vec{\Delta}_{(\Delta x, \Delta y, \Delta z)}<\sqrt{\frac{\left(I_{1}-1\right)\left[{\overrightarrow{R_{1}}}_{(x, y, z)}\right]^{2}}{I_{1}}}-\left[{\overrightarrow{R_{1}}}_{(x, y, z)}\right]^{2},
$$

where

$$
0 \leq \vec{\Delta}_{(\Delta x, \Delta y, \Delta z)},
$$

by referring to the principle of the trigonometric formula as follows:

$$
\begin{aligned}
f(\theta) & =\arccos \left(\frac{\left|{\overrightarrow{R_{1}}}^{2}+\right|{\overrightarrow{R_{2}}}^{2}-\overrightarrow{|\Delta|}^{2}}{2 \overrightarrow{R_{1} \mid} \cdot \mid \overrightarrow{R_{2} \mid}}\right) \\
& =\arccos \left(\frac{{\overrightarrow{R_{1}}}_{(x, y, z)} \cdot\left({\overrightarrow{R_{1}}}_{(x, y, z)}+\vec{\Delta}_{(\Delta x, \Delta y, \Delta z)}\right)}{\left.\left|\overrightarrow{R_{1}}(x, y, z)\right| \cdot \mid{\overrightarrow{R_{1}}}_{(x, y, z)}+\vec{\Delta}_{(\Delta x, \Delta y, \Delta z)}\right) \mid}\right),
\end{aligned}
$$

where $\theta$ is the light vector angle. Finally, on this basis, a mathematical model which uses the varying light vector angle to describe the stability of the gray value is established.

In this mathematical model, we only need to measure the coordinate information and brightness information at the initial position of the oil film; then, it is possible to obtain a range of light vector angular variations for stabilizing the gray value of the fluorescent oil film. 


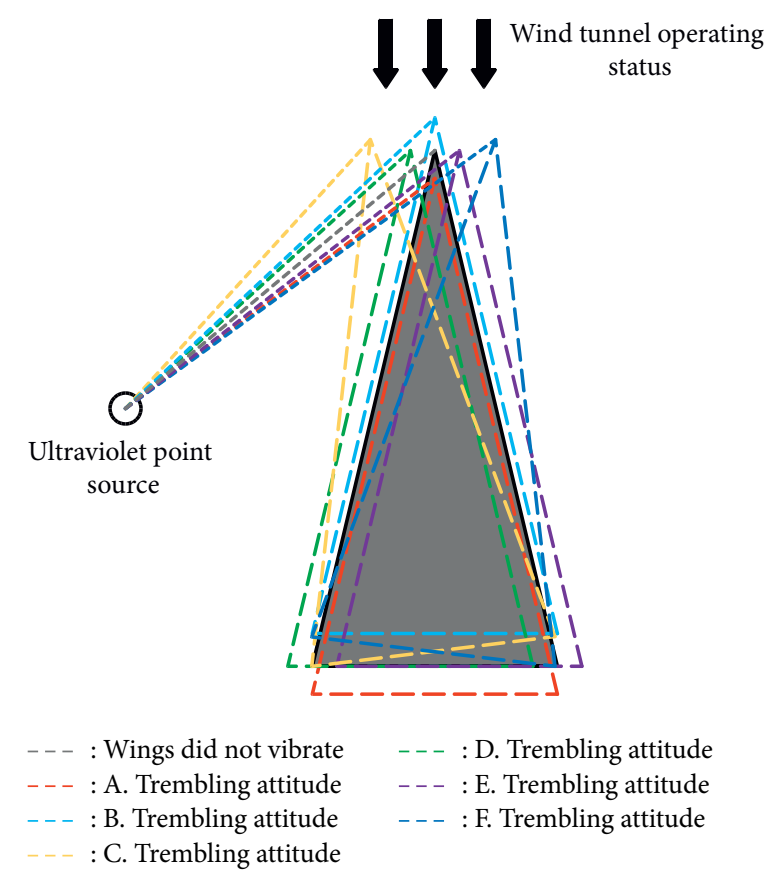

FIgURE 1: Simulation of wing vibration.

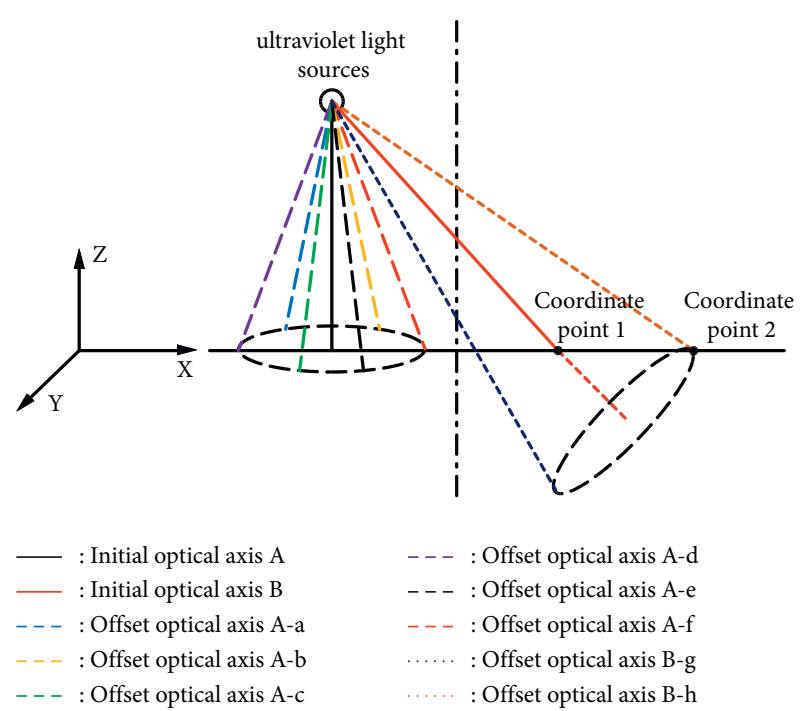

FIgURE 2: Variable UV light vector.

\section{Experiment Operation and Result Analysis}

The position-pose calculation in camera calibration facilitates the transformation of the pixel coordinate system to the world coordinate system, which enables accurate mapping of coordinate points on the pixel plane to the world coordinate system, and this is of great significance in the experiments.

The main technical route of this experiment is shown in Figure 3, where it can be divided into two modules: camera calibration and data processing.

The platform used in the experiment is shown in Figure 4. We use Canon EOS 550D camera during the experiment. Its horizontal resolution and vertical resolution are $72 \mathrm{dpi}$ with a bit depth of 24 . It has the advantages of high resolution, fast acquisition speed, and convenient operation. The model of the UV excitation light source used in the experiment is JZFZ-220/18-001, which has a uniform emission of UV light. The effective power of it is $18 \mathrm{~W} \pm 10 \%$. During the experiment, a $632 \mathrm{~nm}$ optical filter is installed at the camera lens for avoiding the effects of interfering light such as UV or environmental light when the fluorescent oil film is in an excited state. The aim is to achieve an improvement in the accuracy of the gray value.

We use a high-precision sliding platform, as shown in Figure 5, which not only moves freely in the $x$ and $y$ axes but also has a precise scale value attached to it for reference during the actual measurement.

The camera and UV excitation light source will remain fixed at all times during the experiment, which ensures the scientific validity, reliability, and accuracy of the experiment.

3.1. Camera Calibration. The key to obtaining accurate information on the location of the world coordinates corresponding with pixel coordinate points is to complete the transformation between the pixel coordinate system and the world coordinate system [19-21]. The theoretical model for transforming the coordinate system can be expressed as

$$
\lambda\left[\begin{array}{c}
u \\
v \\
1
\end{array}\right]=P_{3 \times 4}\left[\begin{array}{c}
X_{W} \\
Y_{W} \\
Z_{W} \\
1
\end{array}\right] .
$$

Here, we define that the pixel scale factor is $\lambda$, the pixel coordinate is $(u, v)$, and the world coordinate point is $\left(X_{W}, Y_{W}, Z_{W}\right)$.

$$
\left\{\begin{aligned}
M_{1}= & {\left[\begin{array}{lll}
a & r & u_{0} \\
0 & b & v_{0} \\
0 & 0 & 1
\end{array}\right] \bullet\left[\begin{array}{cccc}
f & 0 & 0 & 0 \\
0 & f & 0 & 0 \\
0 & 0 & 1 & 0
\end{array}\right], } \\
M_{2}= & {\left[\begin{array}{ll}
R & T \\
O & 1
\end{array}\right] } \\
& P_{3 \times 4}=M_{1} \bullet M_{2} .
\end{aligned}\right.
$$

In equation (16), $M_{1}$ is the internal camera parameter matrix that determines the relationship between the camera objective and the image plane, and $M_{2}$ is the external parameter matrix which is used to determine the position-pose relationship between these three-dimensional coordinate systems [22, 23].

Considering to make the method of camera calibration simple and effective, Zhang Zhengyou's camera calibration method is used in this experiment. In order to improve the accuracy of the position-pose calculations, distortion correction is applied to the radial and tangential distortion coefficients on the captured images.

Then, the camera calibration is completed by sorting the code points and matching the target points with the same name. The multiple arbitrary position-pose calibration images which we used are shown in Figure 6. The camera 


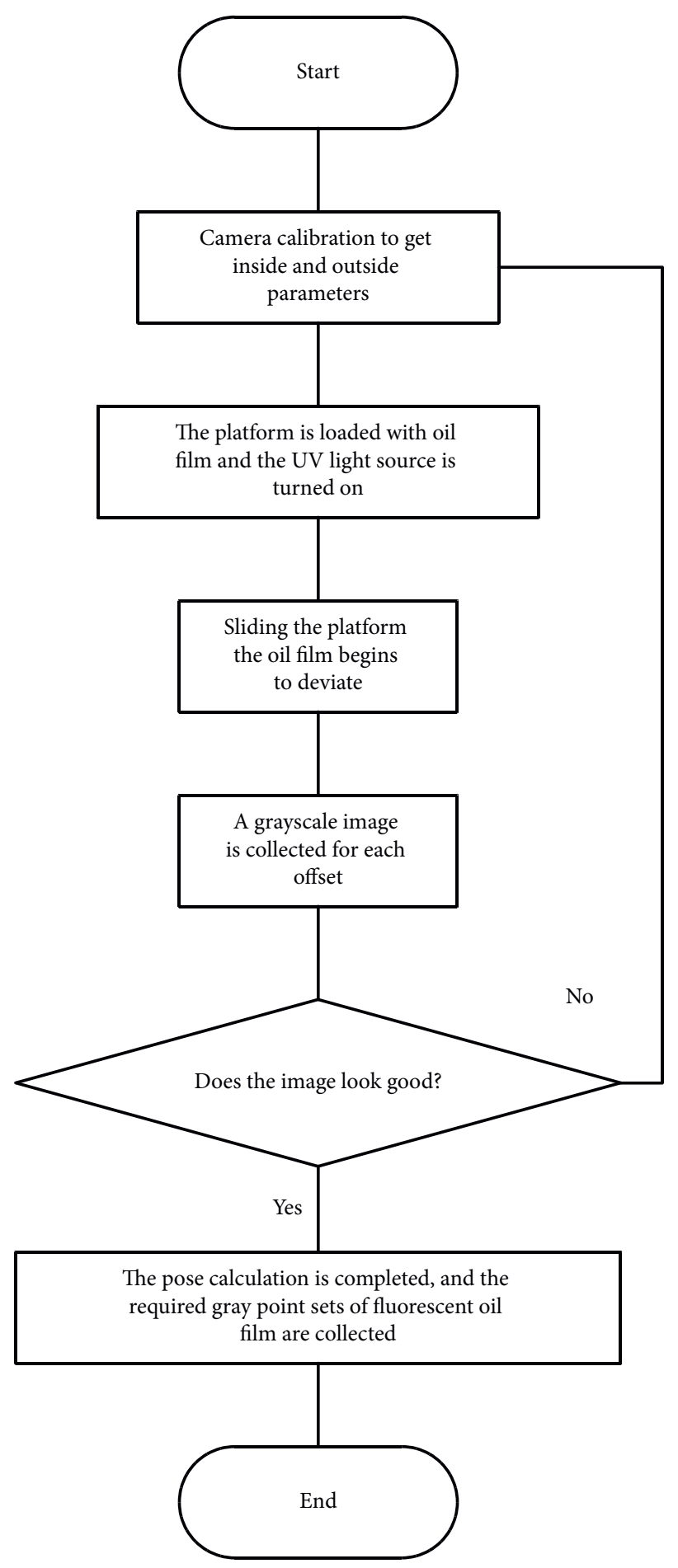

Figure 3: Technical route. 

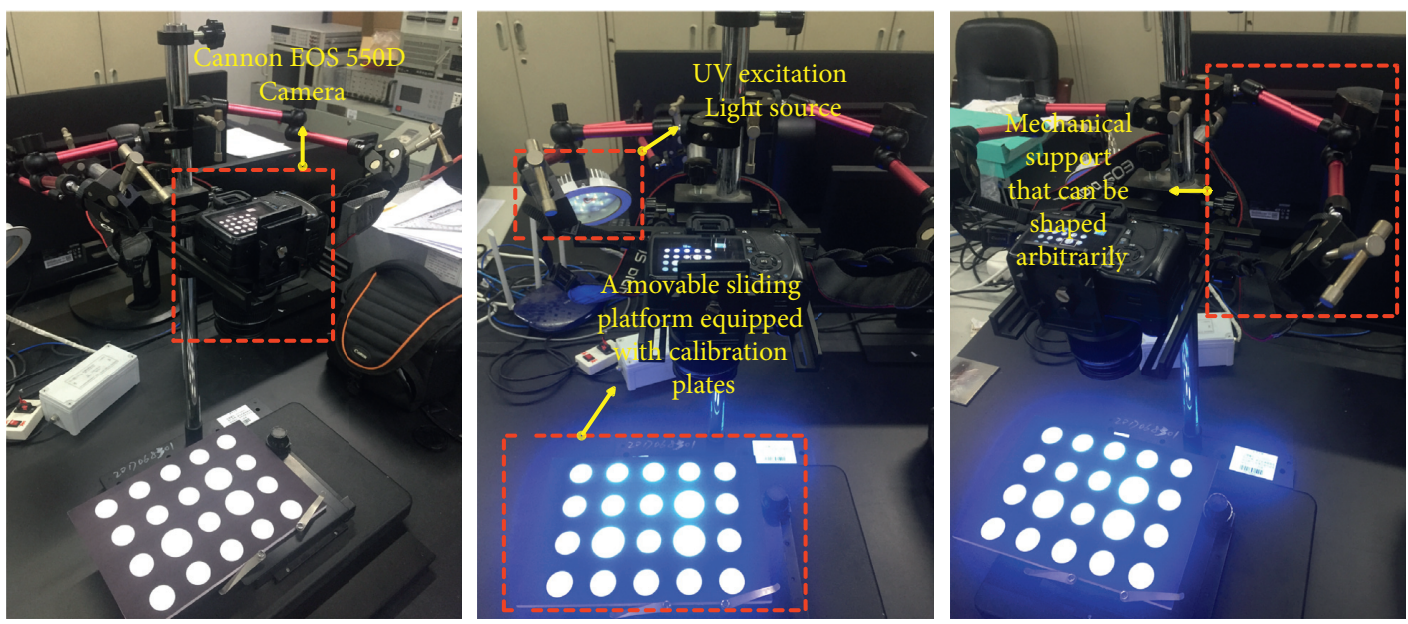

FIGURE 4: Experiment platform.

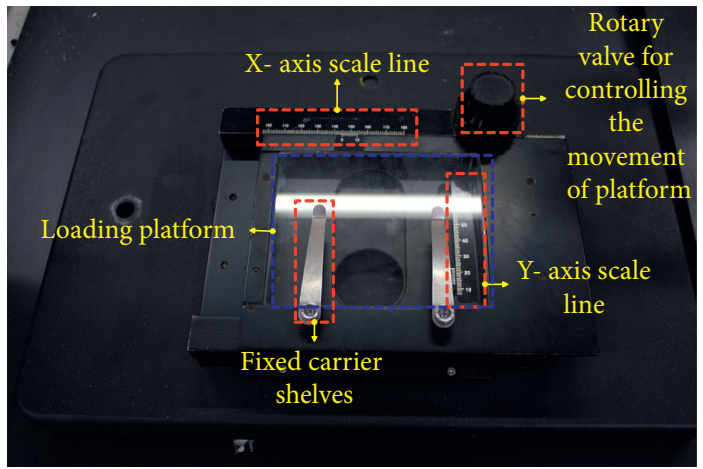

FIGURE 5: High-precision sliding platform.

distortion parameters and internal camera parameters obtained from the experiments are shown in Table 1.

After the camera calibration process is completed, an additional image is taken for decoding, as shown in Figure 7, which is intended to complete the position-pose calculations.

And the matrix of external parameters obtained is shown in the following equation:

$$
\left\{\begin{array}{l}
R=\left[\begin{array}{lll}
\alpha_{1} & \alpha_{2} & \alpha_{3} \\
\beta_{1} & \beta_{2} & \beta_{1} \\
\gamma_{1} & \gamma_{2} & \gamma_{3}
\end{array}\right]=\left[\begin{array}{ccc}
0.9999 & -0.0017 & 0.0053 \\
0.0012 & 0.9962 & 0.0871 \\
-0.0053 & -0.0871 & 0.9962
\end{array}\right], \\
T=-R\left[\begin{array}{l}
X_{s} \\
Y_{s} \\
Z_{s}
\end{array}\right]=\left[\begin{array}{l}
-166.2715 \\
-61.1225 \\
301.8528
\end{array}\right] .
\end{array}\right.
$$

Here, $\alpha_{1}, \alpha_{2}, \alpha_{3}, \beta_{1}, \beta_{2}, \beta_{3}, \gamma_{1}, \gamma_{2}$, and $\gamma_{3}$ are the coefficients obtained by multiplying the matrices of three Euler angles $\phi$, $\omega$, and $\kappa$, respectively. $X_{s}, Y_{s}$, and $Z_{s}$ are the origin coordinates of the camera coordinate system after rotation.

3.2. Data Processing and Result Analysis. In this experiment, a custom-made fixation groove was used to fill the fluorescent oil film, which aimed to avoid diffusion or flow of oil film and to improve the stability of the measurement. The sample of the image taken is shown in Figure 8:

The world coordinate system established during the experiment is shown in Figure 9. To improve the stability of the collected gray value data, pixel values of the measurement points are averaged, and the working distribution of the sampling points is shown in Figure 10.

Grayscale processing of the images is carried out to facilitate the reading of the pixel matrix by MATLAB software. The grid processing of the images enables us to find the exact coordinate values of pixels that will be measured.

In particular, some systematic errors will inevitably occur during the camera calibration, but target points 1 to 10 are all performed under the same camera calibration error, and so the effect of this error factor can be relatively ignored.

The world coordinate system established on the decoded diagram in Figure 7 simply moved in parallel to obtain the world coordinate system, as shown in Figure 9.

The direction of movement is from right to left; then, we set the initial movement coordinates on the $X$-axis scale at $(145,0)$. The picture moving in the same direction and distance is shown in Figure 11, and the measured data are shown in Table 2. In this table, the Metrical data ( $\mathrm{mm}$ ) column indicates the moving distance interval at which the fluorescent oil film moves while the gray value is stable. 

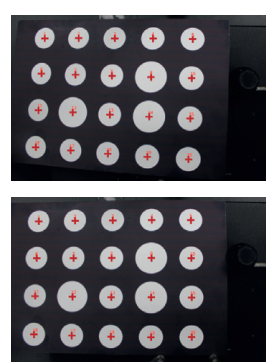
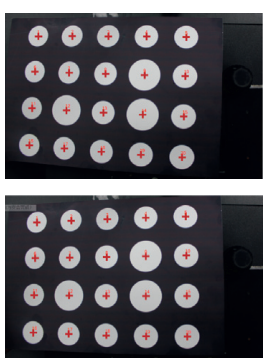
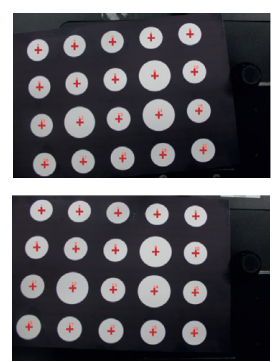
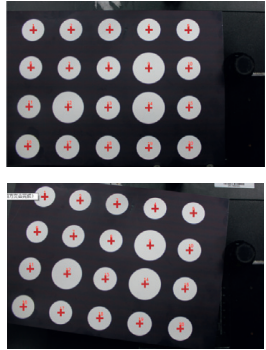
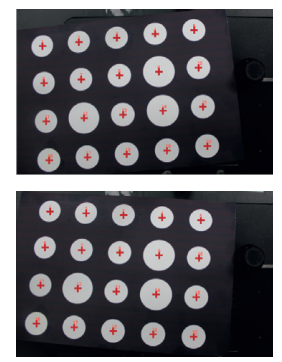

FIGURE 6: Multiple calibrated images of arbitrary positions.

TABLE 1: Camera distortion coefficient and internal parameters.

\begin{tabular}{lccc}
\hline Variable & Radial distortion & Tangential distortion & Intrinsic parameters \\
\hline$k_{1}$ & -0.1469593 & - & - \\
$k_{2}$ & 0.0939691 & - & - \\
$k_{3}$ & 0.0229542 & -0.00257458 & - \\
$p_{1}$ & - & 0.00100651 & - \\
$p_{2}$ & - & - & - \\
$f_{x}, f_{y}$ & - & - & $1759.88,1168.21$ \\
$u_{0}, v_{0}$ & - & - & $17.58(\mathrm{~mm})$ \\
Focal distance & - & & 2722.19 \\
\hline
\end{tabular}

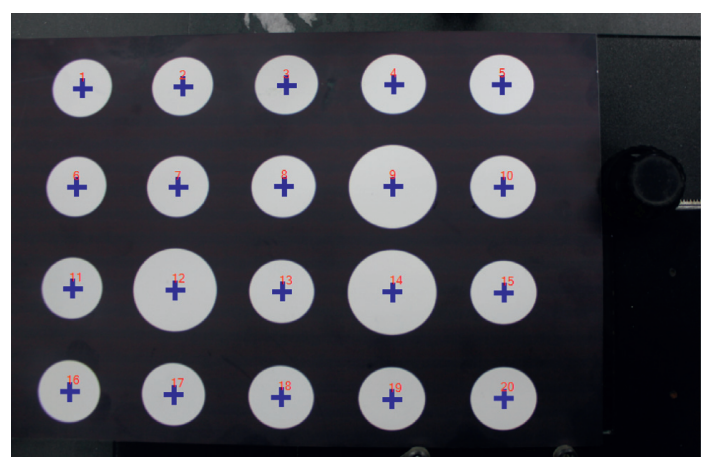

FIgURE 7: Decoding map.

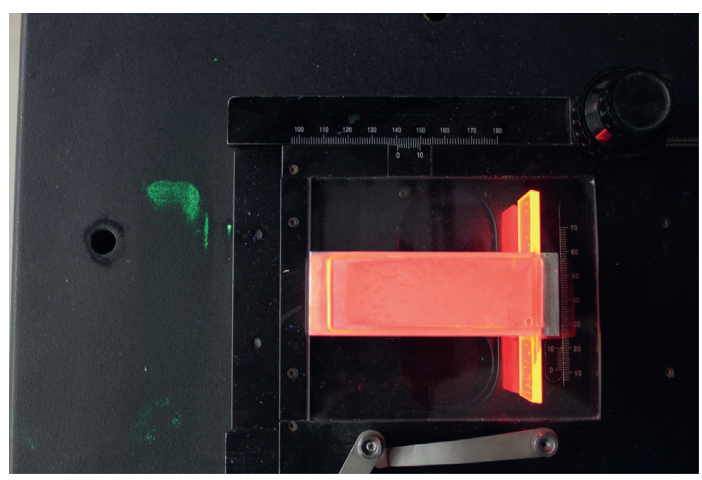

Figure 8: Sample image.

Predict $\theta\left(\times 10^{-7}\right)$ is the maximum critical angle value calculated from the model solution. For example, in the case with measurement point 1 in Table 2 , the gray value of measurement point 1 remains stable as the light vector angle varies between $\left[0^{\circ}, 2.862^{\circ}\right]$. The median value of Metrical data $(\mathrm{mm})$ is used as a reference to facilitate the calculation of errors. Based on the data in Table 2 and validated by experimental analysis, the prediction error of the model 

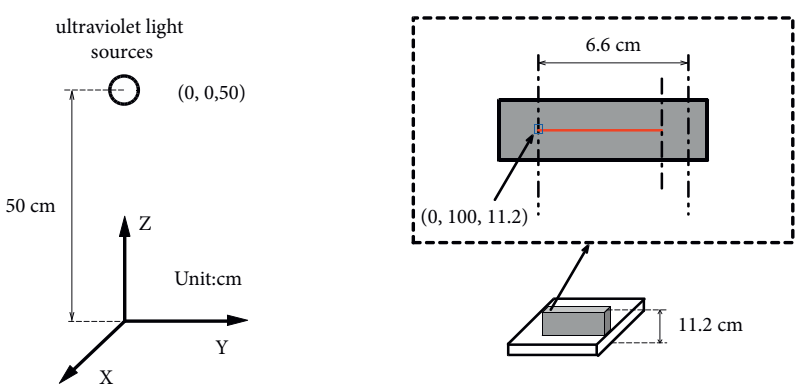

FIgURE 9: Coordination of position information.

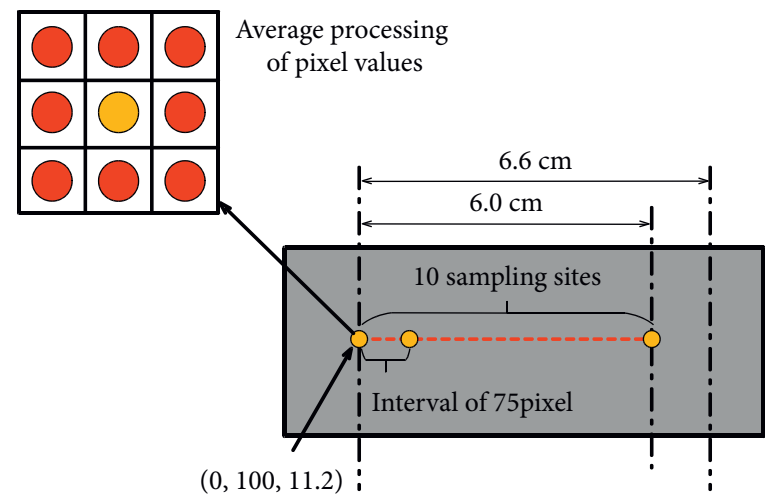

Pixel value to be measured

Adjacent pixel value

Figure 10: Distribution of sampling points. (a) Move $0.5 \mathrm{~mm}$. (b) Move $1.0 \mathrm{~mm}$. (c) Move $1.5 \mathrm{~mm}$. (d) Move $2.0 \mathrm{~mm}$. (e) Move $2.5 \mathrm{~mm}$. (f) Move $3.0 \mathrm{~mm}$. (g) Move $3.5 \mathrm{~mm}$. (h) Move $4.0 \mathrm{~mm}$. (i) Move $4.5 \mathrm{~mm}$. (j) Move $5.0 \mathrm{~mm}$.

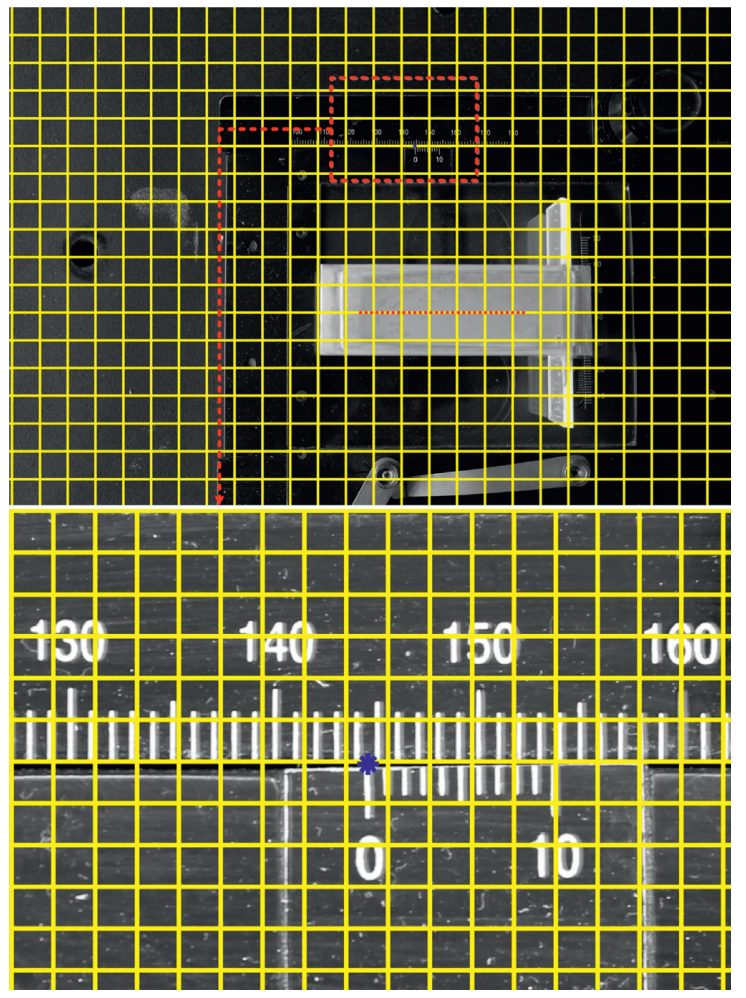

(a)

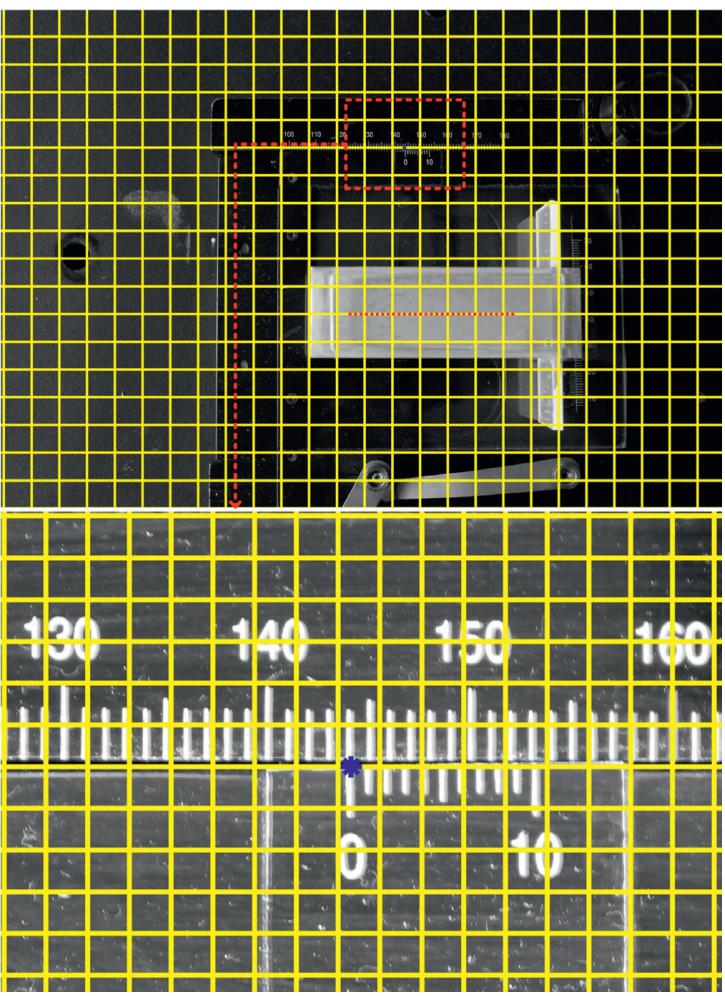

(b)

FIgURE 11: Continued. 


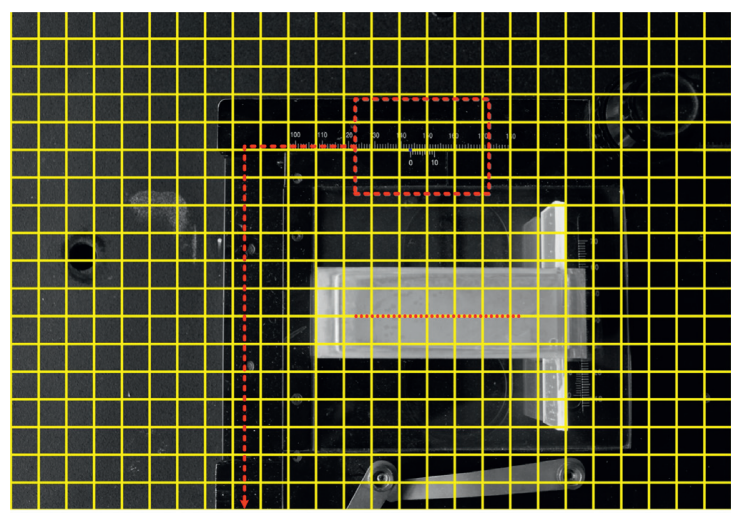

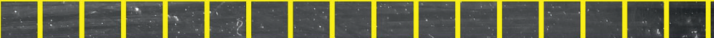
130 190 150 ach H. He tro 100

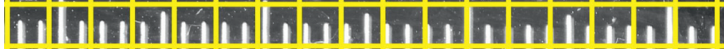

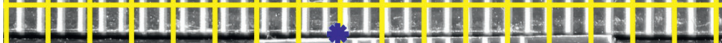

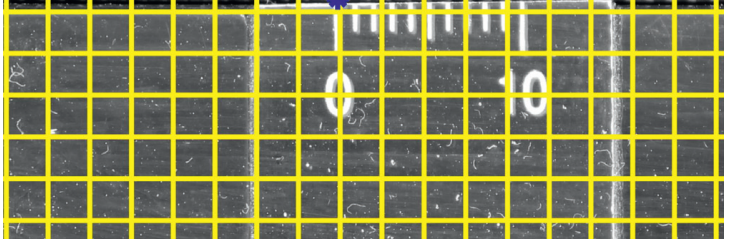
(c)

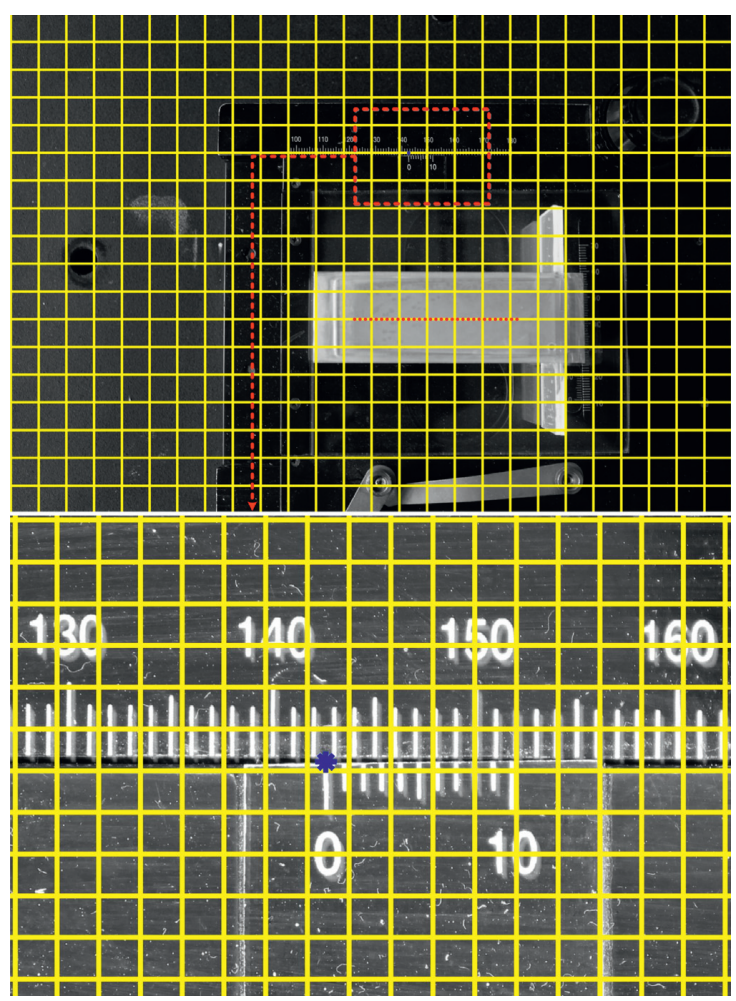

(e)
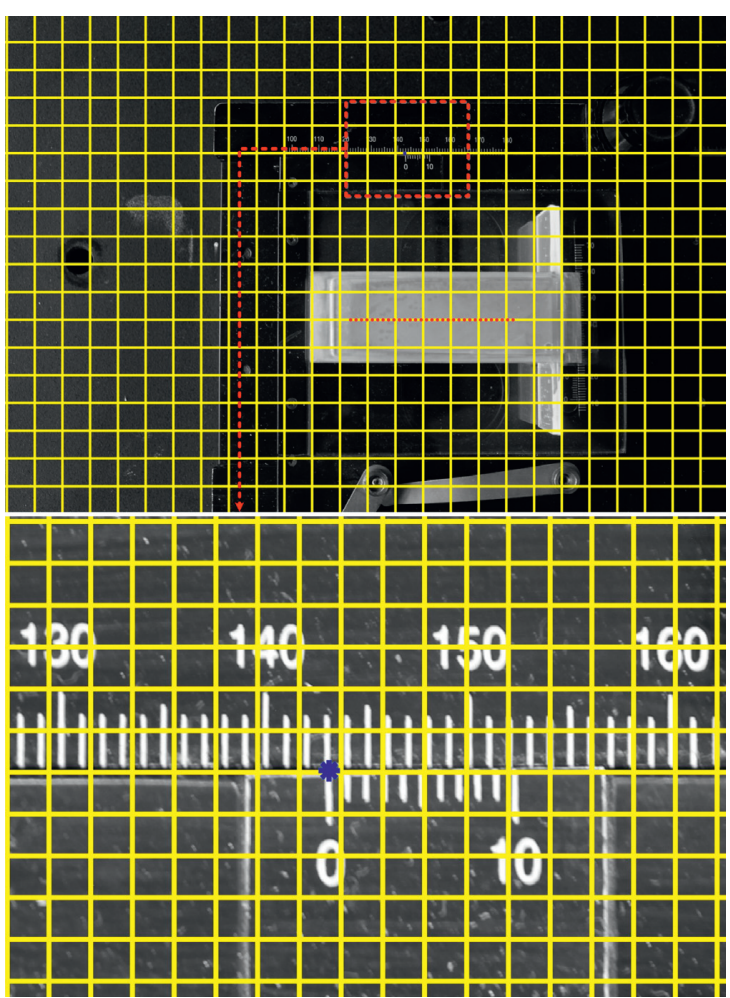

(d)

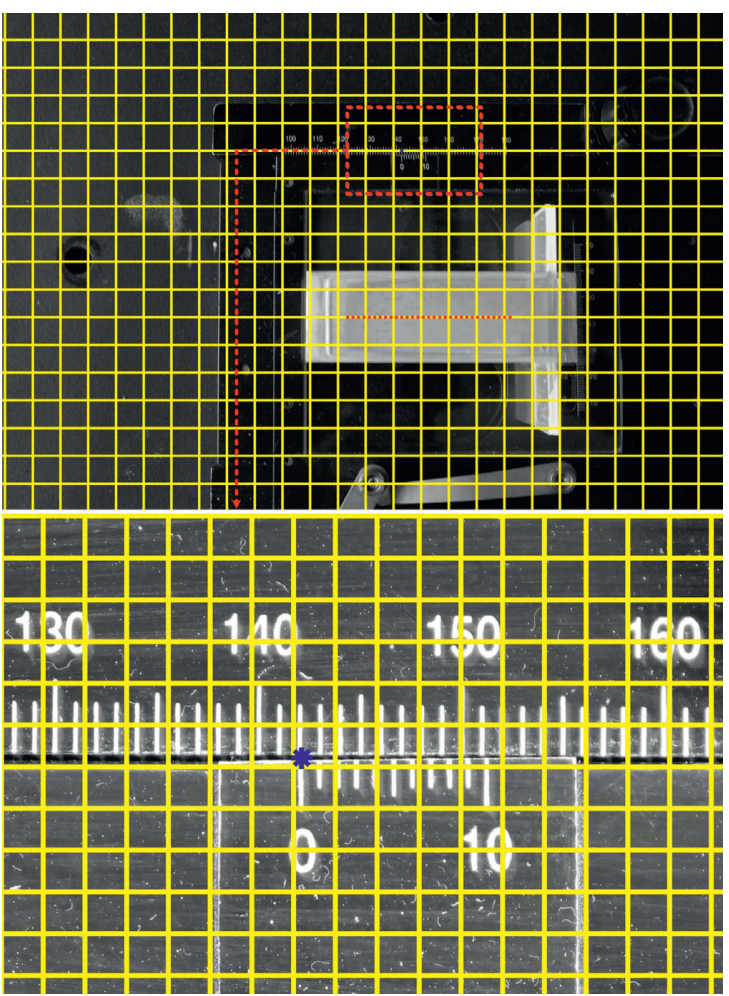

(f)

Figure 11: Continued. 


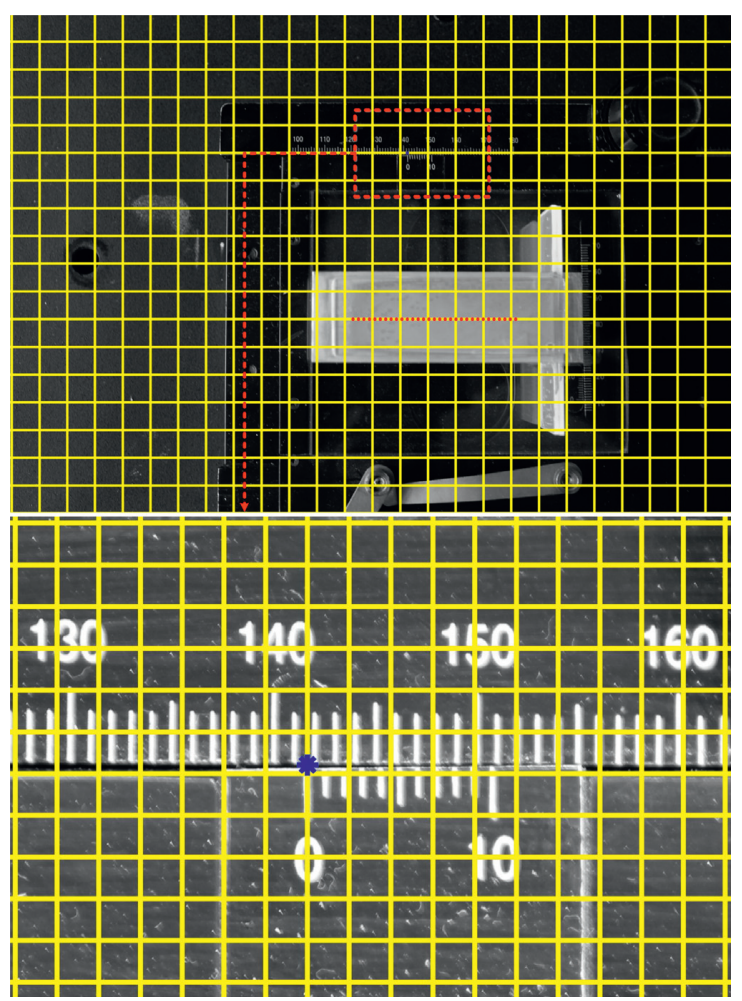

(g)

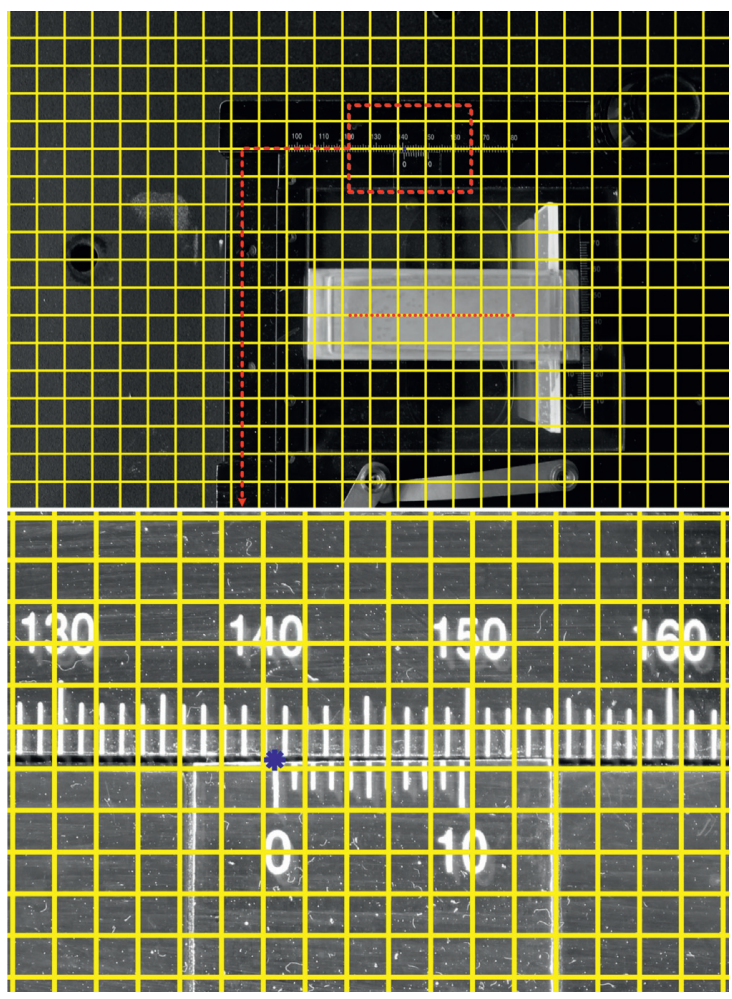

(i)
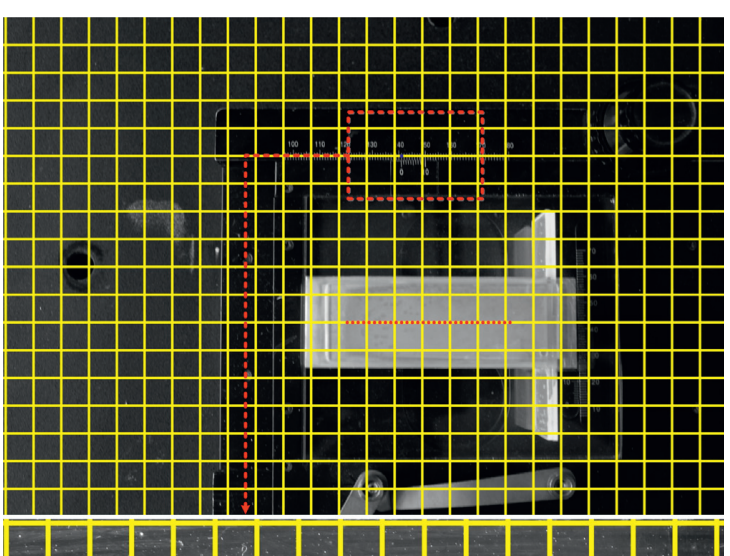
1Rn 1 in $1 \mathrm{kn}$ tan

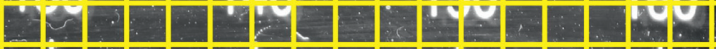

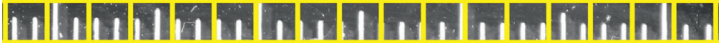

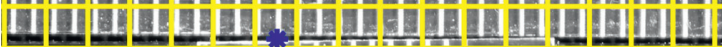

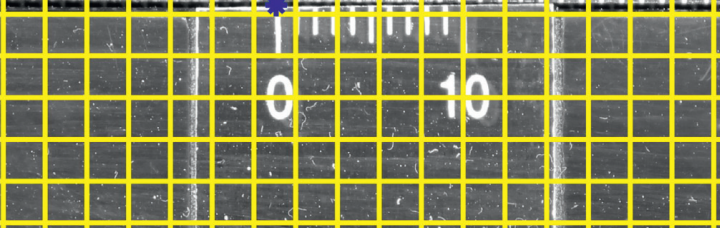

(h)

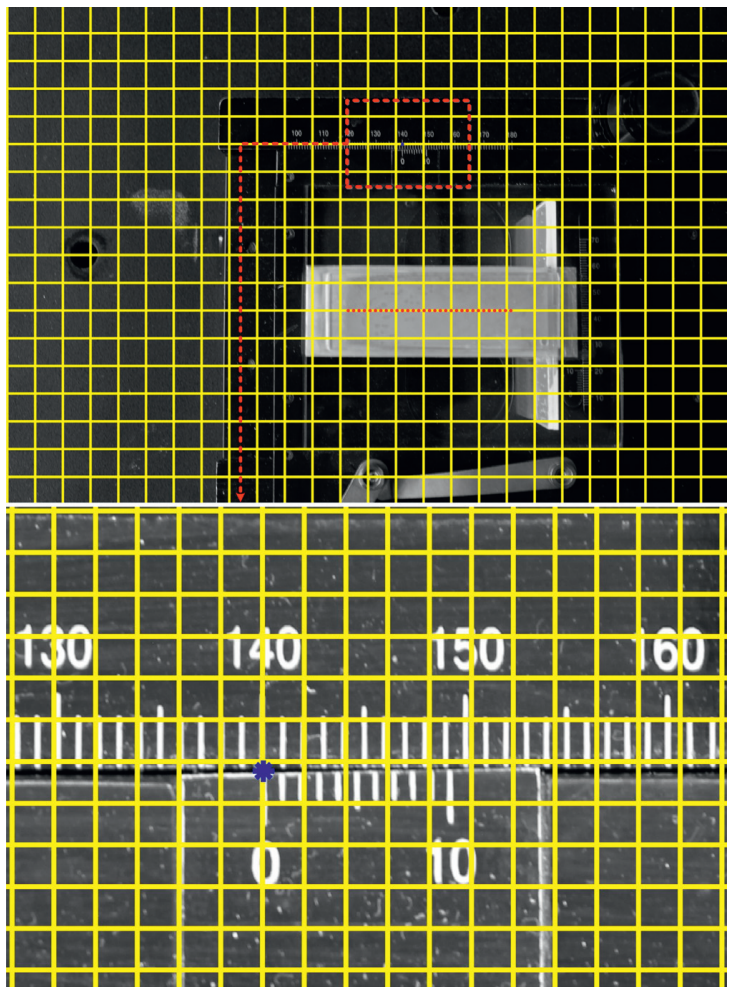

(j)

FIGURE 11: Moving in the same direction and the same distance. 
TABle 2: Moving data in the same direction and distance.

\begin{tabular}{lcccccccccc}
\hline Number & 1 & 2 & 3 & 4 & 5 & 6 & 7 & 8 & 9 & 10 \\
\hline Initial average gray value (pixel) & 161.00 & 166.67 & 166.11 & 164.89 & 164.22 & 162.56 & 160.78 & 159.22 & 157.56 & 154.89 \\
Metrical data $(\mathrm{mm})$ & {$[3,3.5]$} & {$[3,3.5]$} & {$[3,3.5]$} & {$[3,3.5]$} & {$[3.5,4]$} & {$[3,3.5]$} & {$[3,3.5]$} & {$[3.5,4]$} & {$[3.5,4]$} & {$[3.5,4]$} \\
Median of data $(\mathrm{mm})$ & 3.250 & 3.250 & 3.250 & 3.250 & 3.750 & 3.250 & 3.250 & 3.750 & 3.750 & 3.750 \\
Predict $|\vec{\Delta}|(\mathrm{mm})$ & 3.364 & 3.266 & 3.294 & 3.335 & 3.336 & 3.418 & 3.474 & 3.526 & 3.581 & 3.661 \\
Error & $3.51 \%$ & $0.49 \%$ & $1.35 \%$ & $2.62 \%$ & $11.04 \%$ & $5.17 \%$ & $6.89 \%$ & $5.97 \%$ & $4.51 \%$ & $2.37 \%$ \\
Predict $\theta\left(\times 10^{-7}\right)$ & 2.862 & 3.158 & 1.273 & 1.632 & 3.371 & 1.429 & 3.418 & 2.909 & 2.134 & 4.657 \\
\hline
\end{tabular}

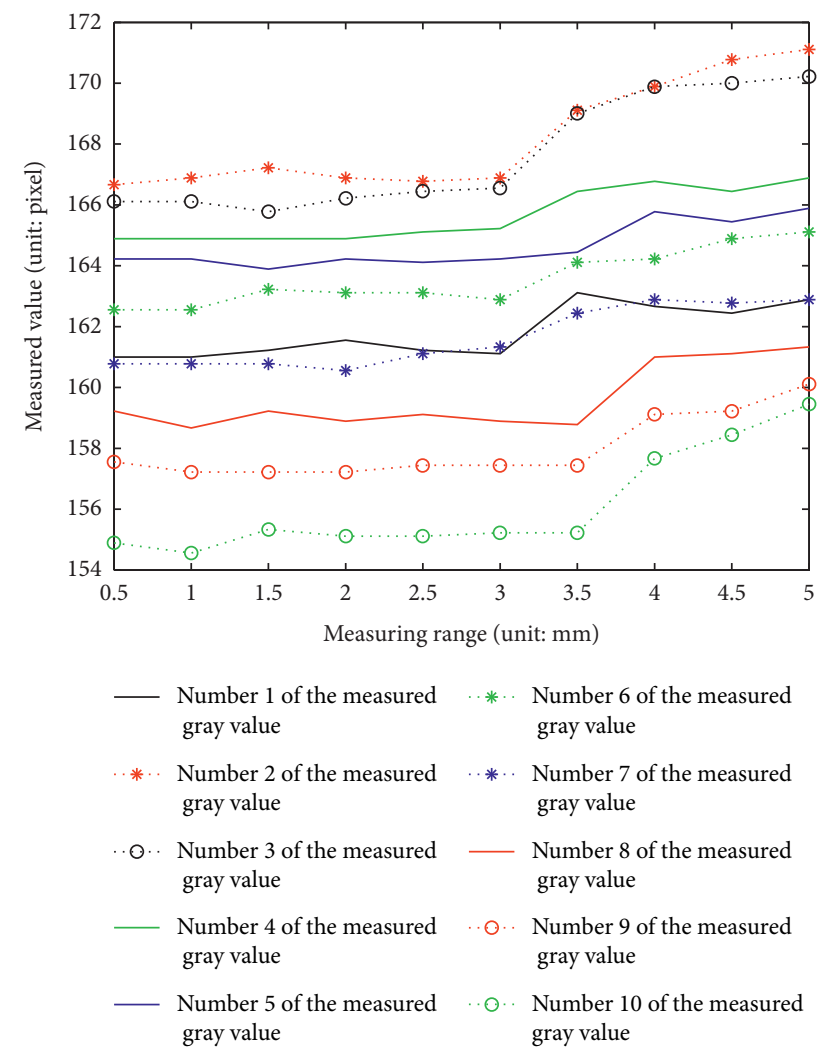

FIGURE 12: Gray value curve distribution of the sampling point.

established in this paper is $4.39 \%$, that is, the prediction accuracy of the model is $95.61 \%$. The distribution of the gray value curves for the sampling points is shown in Figure 12.

We combine Table 2 and Figure 12 for analysis. It can be seen that the fluorescent oil film measurement point is further away from the UV excitation light source. The gray value is low, which conforms to the laws of natural science. The larger error at the measurement point 5 may be due to the fact that fluorescent oil film concentration is higher at this location compared to other areas. So it can absorb more light energy, which changes its gray value and affects the model's prediction here.

\section{Conclusion}

By researching the stability of fluorescent oil film greyscale values under wing vibration conditions, a mathematical theory was derived and analyzed, which was then validated by practical tests. After the model and data analysis, the following conclusions were drawn:
(1) In this paper, a mathematical model for judging the stability of the gray value of the fluorescent oil film is developed based on the background of the vibration conditions of the wing. Within this model, we only need to measure the coordinate information and brightness information from the initial position of oil film to find the vibration range that makes the gray value of the fluorescent oil film stable, and the light vector angle is used in the model to characterize this vibration range, which makes the results more intuitive and concise.

(2) The results were tested through practical experiments. According to the analysis of the experimental data, the prediction accuracy of the mathematical model established in this paper is as high as $95.61 \%$, which reaches the index of practical engineering applications. This will provide a strong vibration range constraint for the global skin-friction measurement of aircraft and exclude the influence of vibration interference on the global skin damage measurement, which has certain practical engineering application significance.

\section{Data Availability}

The data used to support the findings of this study were supplied by Hongiiang Qian under license and so cannot be made freely available. Requests for access to these data should be made to Hongjiang Qian (e-mail: 956312605@ qq.com).

\section{Conflicts of Interest}

The authors declare that they have no conflicts of interest.

\section{Acknowledgments}

This research was supported by the National Natural Science Foundation of China (Nos. 11872069 and 62003278), Key Project of Sichuan Science and Technology Department (No. 2018JY0463), Sichuan University Research Innovation Team "Machine Vision and Intelligent Control" (No. 18TD0024), National Ministry of Education "Chunhui Plan" Scientific Research Project (No. Z2017076), and Xihua University Graduate Innovation Fund (No. YCJJ2021066).

\section{References}

[1] S. A. Woodiga and T. Liu, "Skin friction fields on delta wings," Experiments in Fluids, vol. 47, no. 6, pp. 897-911, 2009. 
[2] H. Qian, X. Dong, and Z. Zhang, "Improved prediction model of gray and thickness of fluorescent oil film based on Hankel matrix," Journal of Aerospace Power, vol. 36, no. 10, pp. 2061-2071, 2021.

[3] Y. Zhang, X. Fang, H. Chen, S. Fu, Z. Duan, and Y. Zhang, "Supercritical natural laminar flow airfoil optimization for regional aircraft wing design," Aerospace Science and Technology, vol. 43, no. jun, pp. 152-164, 2015.

[4] H. Qian, X. Dong, Y. Xu, J. Jiang, and G. Chen, "Fluorescence oil film thickness and gray level identification based on neural network," Journal of Nanjing University of Aeronautics \& Astronautics, vol. 53, no. 3, pp. 470-476, 2021.

[5] T. Liu, S. Woodiga, J. Montefort, K. J. Conn, and L. Shen, "Global skin friction diagnostics in separated flows using luminescent oil," Journal of Flow Visualization and Image Processing, vol. 16, no. 1, pp. 19-39, 2009.

[6] Z. Zhu, Z. Wu, and J. Ding, "Laminar flow control technology and application," Acta Aeronautica et Astronautica Sinica, vol. 32, no. 5, pp. 765-784, 2011.

[7] L. H. Tanner, "A skin friction meter, using the viscosity balance principle, suitable for use with flat or curved metal surfaces (based on thickness measurement)," Journal of Physics E: Scientific Instruments, vol. 10, no. 3, pp. 278-284, 1977.

[8] J. L. Brown and J. W. Naughton, The Thin Oil Film Equation, NASA_AMES Research Center, Washington DC, 1997.

[9] T. Liu, S. Woodiga, and T. Ma, "Skin friction topology in a region enclosed by penetrable boundary," Experiments in Fluids, vol. 51, no. 6, pp. 1549-1562, 2011.

[10] T. Liu, J. Montefort, S. Woodiga, P. Merati, and L. Shen, "Global luminescent oil-film skin-friction meter," AIAA Journal, vol. 46, no. 2, pp. 476-485, 2008.

[11] L. Peng, Research on direct measurement technology of global surface friction stress, Nanjing University of Aeronautics and Astronautics, Nanjing, 2012.

[12] T. Liu and L. Shen, "Fluid flow and optical flow," Journal of Fluid Mechanics, vol. 614, pp. 253-291, 2008.

[13] L. Peng and M. Xiao, "Fluorescent oil film method for global surface friction measurement of wind turbine blades," Journal of Nanjing University of Aeronautics \& Astronautics, vol. 43, no. 5, pp. 581-585, 2011.

[14] H. Zhan, H. Wang, L. Wei, Z. Miao, and C. Pan, "Research of global skin friction measurement based on fluorescent oil film," Acta Aerodynamica Sinica, vol. 34, no. 3, pp. 373-378-403, 2016.

[15] Y. Zou, Z. Zhang, X. Wang, X. Huang, and J. Fan, "Measurement of fluorescent oil film path velocity on the surface of wind tunnel test model," Acta Aeronautica et Astronautica Sinica, vol. 40, no. 6, pp. 92-105, 2019.

[16] D. H. Yu, L. Li, and K. Zhao, "Analysis on transmission of UV radiant energy in rapid prototyping," Advanced Materials Research, vol. 279, pp. 307-312, 2011.

[17] H. Zhou, "Calibration of light beam's direction of point light source probe," Chinese Journal of Scientific Instrument, vol. 25, no. 3, pp. 388-391, 2004.

[18] D. Q. Ling, S Shen, and H. J. Yang, "Measurement of weld defect depth size based on point light source X-ray," Transactions of the China Welding Institution, vol. 21, no. 3, pp. 5-8, 2000.

[19] L. Luo, K. Chen, and J. H. Chen, "Mechanism design method for obtaining a cylinder-like objects pose and position," Advanced Materials Research, vol. 945-949, pp. 1348-1352, 2014.
[20] S.-T. Yee, W. Wan, and J. A. Ventura, "3-D localization and feature recovering through CAD-based stable pose calculation," Pattern Recognition Letters, vol. 22, no. 2, pp. 105-121, 2001.

[21] J. Zhang, J. L. Zhang, H. S. Lei, W. M. Zhao, and C. Doolan, "Calibration method of two-dimensional hot-wire anemometer based on implicit temperature correction," Journal of Aerodynamics, vol. 038, no. 001, pp. 43-47, 2020.

[22] K. Krinkin, K. Chayka, A. Filatov, and A. Filatov, "Autonomous wheels and camera calibration in duckietown project," Procedia Computer Science, vol. 186, pp. 169-176, 2021.

[23] K. Takahashi, D. Mikami, M. Isogawa, A. Kojima, and H. Kimata, "Extrinsic camera calibration of display-camera system with cornea reflections," IEICE Transactions on Information and Systems, vol. E101.D, no. 12, pp. 3199-3208, 2018. 